\title{
Evaluation of Human Mammaglobin as a Biomarker of Circulating Tumor Cells in Breast Cancer and Clinical Verification
}

Chuanguang Xiao

zi bo shi zhong xin yi yuan: Zibo Central Hospital

Xiaohong Wang

Qilu medical college

Shusheng Qiu

zi bo shi zhong xin yi yuan: Zibo Central Hospital

Wenyue Gu ( $\nabla$ alagalaxy@163.com )

Southeast University https://orcid.org/0000-0003-2178-8637

\section{Nano Express}

Keywords: Circulating tumor cells, Breast cancer, Epithelial cell adhesion molecule, Cytokeratin 19, Humanmammaglobin

Posted Date: October 12th, 2020

DOI: https://doi.org/10.21203/rs.3.rs-87537/v1

License: (c) (i) This work is licensed under a Creative Commons Attribution 4.0 International License. Read Full License 


\section{Abstract}

Cytokeratin (CK) is the gold standard marker for the differential diagnosis of epithelial circulating tumor cells (CTC), but the low expression of CK can lead to false negative results.In this study, the specificity and sensitivity of human breast globin (hMAM) as a tumor marker of CTC in peripheral blood of breast cancer patients were analyzed and aim to improve the CTC detection accuracy in breast cancer, enrich the candidate markers for clinical differential diagnosis of breast cancer CTC. EpCAM antibody modified liposome magnetic particles (ELMP) were prepared, and then their physicochemical properties were characterized. ELMP has high physicochemical stability and can efficiently enrich the CTC of epithelial breast cancer. We found hMAM is more consistent with clinical and pathological diagnosis results. To be noted, CK19 combined with hMAM method efficiently distinguish the separated CTC from breast cancer patients, furthermore, the specificity and sensitivity of CTC detection get promoted.

\section{Introduction}

Breast cancer is a type of malignant tumor with a higher risk of metastasis and recurrence[1, 2]. Therefore, using sensitive biomarkers to detect metastasis earlier and using reasonable treatment measures are helpful to improve patient survival[3-6]. Circulating tumor cell (CTC) detection is a viable way for breast cancer diagnosis, staging and transfer risk monitoring[7-9]. Due to the heterogeneity of tumors, breast cancer expresses multiple tumor markers at the same time, so accurate biomarkers are urgently needed for patient management and prognosis[10, 11]. In recent years, methods to detect and separate CTC from different sources of organs have been developed[12, 13]. Current CTC detection techniques include magnetic separation technology based on immune-capture blood CTC, microchip and filter membrane separation method, and improving the convenience and sensitivity of CTC enrichment[14-16]. Epithelial-cell adhesion molecule (EpCAM) plays an important role in carcinogenesis and it is usually overexpressed in most tumor cells of epithelial origin, and not in mesoderm-derived blood cells and lymphocytes. The EpCAMbased separation method have been extensively used in clinical for multiple types of cancer[17-19].

After CTC is separated, immunofluorescence method is used to identify tumor characteristics. Cytokeratin 19 (CK19) CK19 is a typical marker of epithelial cells and CTCs. However, the level of CK19 expression for those CTCs undergo the Epithelial-mesenchymal transition (EMT) process will present low expression level of CK19[20, 21], inevitably brings false negative results[22].Human mammaglobin (hMAM) are tissue-specific markers of breast epithelial tissue and their abnormal expression is closely related to micrometastases[23, 24], hMAM is expressed only in mammary epithelial cells and overexpressed mainly in breast cancer cells[25, 26].

In this study, we prepared EpCAM antibody modified liposome magnetic particles (ELMP) in order to separate CTC from breast cancer clinical blood samples. The CTCs counting results by using CK19 alone and the combined use of hMAM and CK19 as breast cancer CTCs markers independently. We compared the correlation between pathological diagnosis of breast cancer patients and the upon two methods for exploring the clinical diagnostic value of hMAM as a specific tumor marker of CTC in breast cancer patients, aiming to improve the accurate of breast cancer CTCs differential diagnosis.

\section{Materials And Methods}

\section{Materials}


Anti-human EpCAM antibody produced in rabbit was purchased from Sigma-Aldrich; Anti-human CD45-PE produced in mouse was purchased from eBioscience; PE-labeled CK antibody purchased from Johnson \& Johnson Veridex; anti-human hMAM antibody produced in rabbit was purchased from Proteintech; Hoechst 33342 Staining Solution staining solutions was purchased from Beyotime; magnetic nanoparticles was purchased from Nanjing XF NANO; DSPE-PEG ${ }_{2 K}, 1$,2-dioleoyl-sn-glycero-3-phosphocholine (DOPC) and DSPE-PEG $2 K^{-}$Maleimide (DSPE-PEG-Mal) were purchased from Xi'an Ruixi; cholesterol, methylene chloride, and other commonly used reagents purchased from Sinopharm. QDream Antibody Labeling Kit was purchased from Najingbio.

\section{Cell lines}

Human mammary gland cell MCF-10A cells, Cervical cancer Hela cells, breast cancer cell MDR-MB-231, SK-BR-3, MCF-7 and 293T were purchased from ATCC cell bank and kept in the cell lab of Changzheng Hospital; DMEM culture medium, fetal bovine serum and trypsin purchased from Gibco.

\section{Clinical samples collection}

This clinical study was approved by Zibo Central Hospital. All the patient and healthy volunteer consents were written informed consents, and that this was conducted in accordance with the Declaration of Helsinki. Peripheral blood was collected from patient and healthy volunteer, each person was collected two tubes of venous blood, $8 \mathrm{ml}$ per tube at Zibo Central Hospital, including 20 normal blood samples from healthy volunteers, 100 blood samples from breast cancer patient. All breast cancer patients were confirmed by breast ultrasonography and MRI, 60 of the 100 breast cancer patients have complete clinical case information and follow-up records. All blood samples were performed CTC separation and identification within $48 \mathrm{~h}$ after the blood collected in anticoagulant vessel collection.

\section{ELMP preparation}

ELMP was prepared refer to the reverse evaporation method[27]. First of all, DSPE-PEG $2 \mathrm{~K}^{-M A L} 0.5 \mathrm{mg}$ and DSPE$\mathrm{PEG}_{2 \mathrm{~K}} 2.0 \mathrm{mg}$ was dissolved in $2.5 \mathrm{~mL} \mathrm{ddH}_{2} \mathrm{O}$, DOPC $3.0 \mathrm{mg}$, raw magnetic beads $1.0 \mathrm{mg}$ and Chol $2.0 \mathrm{mg}$ was dissolved in $1.5 \mathrm{~mL}$ chloroform, the water phase and the organic phase was mixed, followed by ultrasonic treatment to obtain emulsion. Then liposome solution was obtained under reduced pressure by means of rotary evaporator. Secondly, ELMP was prepared by the method in reference[28, 29], the reaction steps are as follows, 5.0 mg liposome solution was added into $4 \mathrm{~mL}$ HEPES buffer $(50 \mathrm{mM}, \mathrm{pH}$ 6.5), then $40 \mu \mathrm{g}$ EpCAM Antibody solution was added and stirred for $12 \mathrm{~h}$ at room temperature. The reaction mixture was dialyzed in a 3,500 Da dialysis bag for $12 \mathrm{~h}$ at $4{ }^{\circ} \mathrm{C}$ to obtain ELMP.

\section{Characterization of ELMP}

The surface potential and particle size of ELMP was measured by particle size potential analyzer (Zetasizer Nano ZS 90). The morphology of ELMP was observed by transmission electron microscopy (TEM), ELMP was dropped onto the copper mesh and observed after drying. Determination of saturation magnetization of ELMP, after the machines were warmed up, instructions and software prompts were followed to calibrate the machine first, and then the right amount of dry sample of magnetic ball powder was taken to be installed and to start measurements. Intrinsic coercivity $\mathrm{HCJ}$ value can be drawn by a magnetic regression line that can come from the test. The saturation magnetization value of the sample can be obtained by its mass values.

\section{hMAM-QDs was reparented by hMAM antibody labeled with quantum dot}


The rabbit anti-human hMAM antibody was labeled with a quantum dot labeling kit (QDream Antibody Labeling Kit), the brief steps are as follows, $10 \times$ reaction solution and DEionized water were used to dilute the antibody concentration to $2 \mathrm{mg} / \mathrm{ml}$, and the buffer system was $1 \times$ reaction solution; according to the molar ratio of activating reagent: antibody $=20: 1$, the activating reagent was added to the above diluted antibody solution, mixed completely, and incubated for 30 minutes at room temperature; according to the molar ratio of antibody: quantum dots $=2: 1$, the quantum dot solution was added to the above reaction mixture, fully mixed, and incubated at room temperature for 30 minutes, labeled step was end.

\section{hMAM expression and analysis}

The expression of hMAM in tumor tissues was detected by immunostaining. Immunohistochemical diagnosis results of different types of tumor tissues provided by the pathology department of Zibo Central Hospital.

\section{Immunofluorescence analysis of tumor markers}

The expression of hMAM in different cell lines was detected by immunostaining, take the cell culture slides into a 24-well plate, plate MCF-10A or tumor cells at a density of 20,000/well, wash twice with PBS after overnight attachment, fix with 4\% paraformaldehyde for 20 min, and wash with PBS twice. Add Hoechst 33342 and CK19-FITC or Hmam-QDs to stain for 15 min, wash twice with PBS, invert the slide on the glass slide covered with the mounting solution, and observe under the laser confocal microscope (Olympus FV10i).

Flow cytometry was used to detect the immunostaining of breast cancer cells by CK19 and hMAM at the same time. MDA-MB-231 cells were plated at a density of 80,000/well in a 12-well plate, and CK19-FITC and hMAM- were added after overnight adhesion. QDs were used for $1 \mathrm{~h}$, washed twice with PBS, digested with trypsin, centrifuged at $1000 \mathrm{rpm}$ for $5 \mathrm{~min}$, collected cells, resuspended with $300 \mu \mathrm{L}$ of PBS containing $1 \%$ FBS, and analyzed by flow cytometry.

\section{Process of isolation and identification breast cancer CTC}

The steps for CTC separation and identification of breast cancer were as follows: (1) collect 7.5 ml blood of breast cancer patients in an anticoagulant tube and centrifuge it at $1000 \mathrm{r} / \mathrm{min}$ for $10 \mathrm{~min}$;(2) take the pelagic liquid and place it in EP tube, add the equivalent amount of PBS and mix it thoroughly;(3) add the immune lipid magnetic sphere $30 \mu \mathrm{L}$, incubate it at room temperature for $30 \mathrm{~min}$, and mix it every $5 \mathrm{~min}$;(4) The EP tube was inserted into the magnetic separation rack for 15 min to absorb and discard the superfluid; (5) wash it once in PBS;(6) Hoechst 33342 staining solution $30 \mu \mathrm{L}$, anti-CK19-FITC staining solution $10 \mu \mathrm{L}$, and anti-CD45-PE staining solution $10 \mu \mathrm{L}$ or hMAM-QDs staining solution $10 \mu \mathrm{L}$ was added to blend and stain for $15 \mathrm{~min}$;(7) wash with double steam water once;(8) $30 \mu \mathrm{L} \mathrm{ddH_{2 }} \mathrm{O}$ was added to the EP tube and resuspended, the droplets were evenly coated on the anti-slide slide. Then, the sample is observed and counted under a fluorescence microscope until the droplets dry in the dark.

\section{CTC isolation efficiency analysis of ELMP}

Breast cancer cells MDA-MB-231 was used as CTC model to study the CTC separation efficiency in use of ELMP. Different amount $(10,20,50,100,200)$ MDA -MB-231 cells were added into $7.5 \mathrm{~mL}$ anticoagulant venous blood separately, the subsequent CTC separation and identification steps was carried out according to the above steps.

\section{Clinical study on isolation and identification of circulating tumor cells}


ELMP was used to separate CTC from 120 peripheral blood samples (20 normal and 100 patient samples), Hoechst 33342, anti-CK19-FITC, and PE-labeled CD45 antibody were used for the separated CTC identification in single tumor marker method. Hoechst 33342, anti-CK19-FITC, and hMAM-QDs were used for the separated CTC identification in double tumor marker method. The CTC positive determination criteria are greater than 5 in $7.5 \mathrm{~mL}$ blood.

\section{Statistical Analyses}

Data were analyzed using descriptive statistics, for multiple comparisons, a one-way ANOVA test was performed, and Data are expressed as mean \pm standard deviation (S.D.) derived averaged across three to at least three independent measurements. Comparisons among different groups were performed by Chi-square tes. Differences were considered as significant and the null hypothesis of no difference among treatment groups was rejected for $P$ values $<0.05$

\section{Results And Discussion}

\section{Preparation and characterization of ELMP}

ELMP was prepared by indirect method (Fig. 1), the antibodies were modified on the surface after the preparation of the liposome magnetic particles, raw $\mathrm{Fe}_{3} \mathrm{O}_{4}$ beads were embedded inside of the liposome, and EpCAM antibodies were modified outside the liposome.

As shown in Fig. 2A, the average diameter of ELMP was $90.1 \pm 0.84 \mathrm{~nm}$ (in $\mathrm{ddH}_{2} \mathrm{O}$ and at $25^{\circ} \mathrm{C}$ ), with a polydispersity index of 0.08. Surface Zeta potential of ELMP was $16.7 \pm 0.32 \mathrm{mV}$ (Figure $2 \mathrm{~B}$ ). Transmission electron microscope results was shown in Fig. 2C, the diameter of ELMP was around $30 \sim 90 \mathrm{~nm}$ in dehydration state. The magnetizing curve under $300 \mathrm{~K}$ temperature is shown in Fig. 2D, the result showed that maximum saturated magnetization of the $\mathrm{Fe}_{3} \mathrm{O}_{4}$ raw beads was $43.5 \mathrm{Am}^{2} / \mathrm{kg}$, and the saturated magnetization of ELMP was 31.3 $\mathrm{Am}^{2} / \mathrm{kg}$, accounting for $72.0 \%$ of the saturated magnetization of the $\mathrm{Fe}_{3} \mathrm{O}_{4}$ raw beads, this suggesting that liposome wrapped the surface of the $\mathrm{Fe}_{3} \mathrm{O}_{4}$ raw beads. The solid content of ELMP was $2.8 \pm 0.15 \mathrm{mg} / \mathrm{mL}$, which fully complied with the test requirements, so the antibody and lipid components were distributed around the magnetic NPs, thereby reducing the saturated magnetization.

\section{hMAM expression analysis in breast cancer tissue}

The expression of hMAM in benign and malignant breast cancer tissues was analyzed by immunohistochemistry. As shown in Fig. 3A, the expression of hMAM in low-grade benign breast cancer tissues was very low, and the expression of hMAM in high-grade benign tumors increased slightly, however the expression of hMAM in malignant breast cancer tissues was high. Figure 3B shows the pathological results of four common malignant tumors, we can clearly find that threr was almost no hMAM expression in other tumor tissues. At the same time, it can be seen from Fig. 3 that high-grade breast cancer cells are highly infiltrated, and the expression level of CK19 is high. CK19 has a higher expression level in both breast and other cancer tissues, so CK19 is a common marker of epithelial, not breast Cancer specific markers. Thus, hMAM with a significant distribution characteristic in breast tissue.

\section{hMAM expression analysis in breast cancer cells}

In order to further investigate the expression of hMAM in tumor cells, several breast cancer cells commonly used in the laboratory were selected and immunofluorescence antibody staining was used to detect the expression of CK19 and hMAM. As shown in Fig. 4, CK19 expression was positive in Hela cells of cervical cancer and three types of 
breast cancer cells, but hMAM expression was negative in Hela cells, weak in McF-7 cells, strong in SK-BR-3 and MDA-MB-231 cell.Similar to the histopathological results, CK19 was widely co-expressed in epithelial cell carcinoma, but hMAM expression was negative, and THE hMAM expression level of MDA-MB-231 was relatively high.

\section{Flow cytometry analysis of MDA-MB-231 cells by CK19-FITC combined with hMAM-QDS staining}

In order to analyze the positive ratio of double-positive cells with simultaneous immunofluorescence staining of CK19 and hMAM in MDA-MB-231 cells, we performed double-fluorescence staining analysis by flow cytometry. As shown in Fig. 5, 96.7\% of MDA- MB-231 cells were double positive for immunofluorescence staining of CK19 and hMAM. Therefore, MDA-MB-231 can be used as a cell model for the evaluation and evaluation of CTC recovery of ELMP.

\section{CTC recovery experiment}

Taking MDA-MB-231, MCF-7 and 293T as the CTC model, as shown in Fig. 6A, the CTC separation and capture performance of ELMP was analyzed through 5 sets of gradient number of CTC recovery efficiency tests. For MDAMB-231 the average recovery rate was $>90 \%$, and the average recovery rate of MCF-7 was close to MDA-MB-231. But very low recovery of 293T,this could attributable to the $n$ on-epithelial origin of 293T cells. The recovering efficiency of MDA-MB-231 cells showed a good linear distribution, $\mathrm{R}^{2}=0.9984$ (Fig. 6B). The above experimental results indicate that ELMP can efficiently capture the CTC in anticoagulated blood. The recovered CTC has a good linear relationship with the initial CTC input in the blood. The high recovery efficiency can be used in clinical research.

\section{Isolation and identification of CTC in peripheral blood}

By magnetic separation, CTC separation of breast cancer clinical blood samples was carried out by means of ELMP. Immunofluorescence staining was performed on the separated cells by two methods, and fluorescent micrographs were taken for statistical analysis of CTC. Observed by fluorescence microscope, the CTC results identified by the two methods are shown in Fig. 7.

Figure 7A shows that CK19-FITC stains green, cell nucleus stains blue, and CD45 staining is negative to determine breast cancer CTC. Figure 7B shows that CK19-FITC stained green, nuclei stained blue, and hMAM-QDs stained cells showing red fluorescence were judged as breast cancer cells. The CTCs that were positive in both determination methods showed large cells, a high proportion of nuclei, and irregular cell morphology, which was consistent with the CTC morphological consensus.

\section{Comparative analysis of clinical results}

CTC was isolated from the blood samples of 20 healthy volunteers and 100 breast cancer patients. Two methods were used for CTC identification. The results are shown in Table 1 after statistics. The CTC statistical results of 20 healthy volunteers showed that 1 case of CTC was misdiagnosed as positive with the single label method, and the positive rate was $5 \%$. There was no CTC misdiagnosis with the single label method, and the positive rate was $0 \%$. There was no significant difference in statistical results $(p=0.311)$. The CTC test results of clinical blood samples of 100 breast cancer patients showed that the CTC positive rate using the single tumor marker method was $82 \%$, and the CTC positive rate using the double tumor marker method was $92 \%$. The statistical results of the two methods were significantly different $(p=0.036)$. The ROC curve was drawn based on the CTC statistical results of 120 clinical blood samples, and the specificity and sensitivity of the two methods were compared. As shown in Fig. 8 and Table 2 , the distribution of the area under the curve of the single tumor marker method at the $95 \%$ confidence 
interval was $0.819 \sim 0.039$, the area under the curve is 0.889 , the distribution of the area under the double tumor marker method in the $95 \%$ confidence interval is 0.908 to 0.987 , and the area under the curve is 0.96 . The above results indicate that the use of the double standard method can reduce the false positives of CTC identification results. And the probability of false negatives, improve detection accuracy.

Table 1

CTC detection results of 20 healthy volunteers and 100 breast cancer patients.

\begin{tabular}{|c|c|c|c|c|c|c|c|c|c|}
\hline \multirow[t]{2}{*}{ Characteristics } & \multirow[t]{2}{*}{ Cases } & \multicolumn{3}{|c|}{ Single tumor marker method } & \multicolumn{3}{|c|}{ Double tumor marker method } & \multirow[t]{2}{*}{$x^{2}$} & \multirow[t]{2}{*}{$p$} \\
\hline & & Positive & Negative & $\begin{array}{l}\text { Positive } \\
\text { rate }\end{array}$ & Positive & Negative & $\begin{array}{l}\text { Positive } \\
\text { rate }\end{array}$ & & \\
\hline $\begin{array}{l}\text { Healthy } \\
\text { volunteers }\end{array}$ & 20 & 19 & 1 & $5 \%$ & 20 & 0 & $0 \%$ & 1.026 & 0.311 \\
\hline $\begin{array}{l}\text { Cancer } \\
\text { patients }\end{array}$ & 100 & 82 & 18 & $82 \%$ & 92 & 8 & $92 \%$ & 4.421 & 0.036 \\
\hline
\end{tabular}

Table 2

The ROC curve characteristic of CTC detection by two methods.

\begin{tabular}{|llll|}
\hline Variable & AUC & SE $^{\mathrm{a}}$ & $\mathbf{9 5 \%} \mathbf{C l}^{\mathrm{b}}$ \\
\hline Single tumor marker method & 0.889 & 0.0287 & 0.819 to 0.939 \\
\hline Double tumor marker method & 0.96 & 0.0136 & 0.908 to 0.987 \\
\hline a DeLong et al., 1988 & & & \\
\hline b Binomial exact & & & \\
\hline
\end{tabular}

The clinical diagnosis results of 100 breast cancer patients were statistically summarized to analyze the characteristic relationship between CTC test results and pathological diagnosis. The analysis results were shown in Table 3, which showed that there was no correlation between CTC detection results and age and pausimenia, but close correlation with blood metastasis, Lymphatic metastasis, $\mathrm{P}<0.05$. There was a significant difference between the positive CTC and TNM stage by the double-standard method $(p=0.019<0.05)$. However, there was no significant difference between the positive results of CTC and TNM staging by single tumor marker method $(p=0.205>0.05)$. TNM stage is an important indicator of tumor. The higher the stage, the higher the risk of metastasis in theory and the corresponding CTC test result should be positive. Therefore, the double tumor marker method can improve the accuracy of CTC test for breast cancer, with higher consistency with the pathological test results. 
Table 3

Relationship between positive rate and clinicopathological characteristics by two methods.

\begin{tabular}{|c|c|c|c|c|c|c|c|c|}
\hline \multirow[t]{3}{*}{ Characteristics } & & \multirow[t]{3}{*}{ Cases } & \multicolumn{3}{|c|}{$\begin{array}{l}\text { Single tumor marker method } \\
(35)\end{array}$} & \multicolumn{3}{|c|}{$\begin{array}{l}\text { Double tumor marker method } \\
(40)\end{array}$} \\
\hline & & & & $x^{2}$ & $\mathrm{p}$ & & $x^{2}$ & $\mathrm{p}$ \\
\hline & & & positive & & & positis & & \\
\hline \multirow[t]{2}{*}{ Age } & 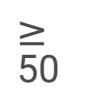 & 17 & 14 & \multirow[t]{2}{*}{0.331} & \multirow[t]{2}{*}{0.565} & 14 & \multirow[t]{2}{*}{1.182} & \multirow[t]{2}{*}{0.277} \\
\hline & $\nabla 50$ & 28 & 21 & & & 26 & & \\
\hline \multirow[t]{2}{*}{ Pausimenia } & Yes & 18 & 12 & \multirow[t]{2}{*}{2.143} & \multirow[t]{2}{*}{0.143} & 15 & \multirow[t]{2}{*}{0.938} & \multirow[t]{2}{*}{0.333} \\
\hline & No & 27 & 23 & & & 25 & & \\
\hline \multirow[t]{2}{*}{ TNM stage } & $\nabla \sim \nabla$ & 15 & 10 & \multirow[t]{2}{*}{1.607} & \multirow[t]{2}{*}{0.205} & 11 & \multirow[t]{2}{*}{5.513} & \multirow[t]{2}{*}{0.019} \\
\hline & $\nabla \sim \nabla$ & 30 & 25 & & & 29 & & \\
\hline \multirow{2}{*}{$\begin{array}{l}\text { Pathological } \\
\text { differentiation }\end{array}$} & High & 30 & 27 & \multirow[t]{2}{*}{7.778} & \multirow[t]{2}{*}{0.006} & 29 & \multirow[t]{2}{*}{5.513} & \multirow[t]{2}{*}{0.019} \\
\hline & Low & 15 & 8 & & & 11 & & \\
\hline \multirow[t]{2}{*}{ Distant metastasis } & Yes & 29 & 27 & \multirow[t]{2}{*}{11.084} & \multirow[t]{2}{*}{$<0.001$} & 29 & \multirow[t]{2}{*}{10.195} & \multirow[t]{2}{*}{$<0.001$} \\
\hline & No & 16 & 8 & & & 11 & & \\
\hline \multirow[t]{2}{*}{ Lymphatic metastasis } & Yes & 33 & 28 & \multirow[t]{2}{*}{7.305} & \multirow[t]{2}{*}{0.007} & 32 & \multirow[t]{2}{*}{8.182} & \multirow[t]{2}{*}{0.004} \\
\hline & No & 12 & 6 & & & 8 & & \\
\hline
\end{tabular}

\section{Conclusions}

In this study, EpCAM antibody modified liposome magnetic particles (ELMP) was successfully assembled with EpCAM antibodies modified on the surface after the preparation of the liposome magnetic particles, raw $\mathrm{Fe}_{3} \mathrm{O}_{4}$ beads were embedded inside of the liposome. In simulated recovery experiment, more than $90 \%$ of CTC was enriched by ELMP, demonstrated a high recovery efficiency. The area under the ROC curve for the differential diagnosis of CTC using combined with CK19 and hMAM method was 0.96, simultaneously, double tumor marker method is more consistent with clinical and pathological diagnosis results. Clinical results show that hMAM is a highly specific marker for the diagnosis of breast cancer, the sensitivity of detection is improved. This study proposals human breast globin as a tumor marker for breast cancer CTC, enrich the candidate markers for clinical differential diagnosis of breast cancer CTC, provides a method for rapid detection and precise diagnosis of breast cancer by a liquid biopsy, with the advantages of noninvasive, convenient and repeatable.

\section{Declarations}

\section{Authors' contributions}

Wenyue Gu and Shusheng Qiu contributed to the conception and design the study; Chuangguang Xiao and Xiaohong Wang performed the experimental work; Chuangguang Xiao and Wenyue Gu contributed to the sample selection and data analysis. Wenyue Gu wrote the manuscript. All authors read and approved the final manuscript. 


\section{Acknowledgments}

Thanks to the help in sorting out the breast cancer pathology pathological data of Pathology department of Zibo Central Hospital.

\section{Disclosure}

The authors report no conflicts of interest in this work.

\section{References}

1. Dittmer J (2017) Mechanisms governing metastatic dormancy in breast cancer. Semin Cancer Biol 44:72-82

2. Wagenblast $E$, Soto $M$, Gutiérrez-Ángel S et al (2015) A model of breast cancer heterogeneity reveals vascular mimicry as a driver of metastasis. Nature 520:358-362

3. Cristofanilli M, Turner NC, Bondarenko I et al (2016) Fulvestrant plus palbociclib versus fulvestrant plus placebo for treatment of hormone-receptor-positive, HER2-negative metastatic breast cancer that progressed on previous endocrine therapy (PALOMA-3): final analysis of the multicentre, double-blind, phase 3 randomised controlled trial. Lancet Oncol 17:425-439

4. Franchino F, Rudà R, Soffietti R (2018) Mechanisms and Therapy for Cancer Metastasis to the Brain. Front Oncol 8:161

5. Ilié M, Szafer-Glusman E, Hofman V et al (2018) Detection of PD-L1 in circulating tumor cells and white blood cells from patients with advanced non-small-cell lung cancer. Ann Oncol 29:193-199

6. Mateo J, Fizazi K, Gillessen S et al (2019) Managing Nonmetastatic Castration-resistant Prostate Cancer. Eur Urol 75:285-293

7. Bidard FC, Proudhon C, Pierga JY (2016) Circulating tumor cells in breast cancer. Mol Oncol 10:418-430

8. Gkountela S, Castro-Giner F, Szczerba BM et al (2019) Circulating Tumor Cell Clustering Shapes DNA Methylation to Enable Metastasis Seeding. Cell 176:98-112.e114

9. Rossi G, Mu Z, Rademaker AW et al (2018) Cell-Free DNA and Circulating Tumor Cells: Comprehensive Liquid Biopsy Analysis in Advanced Breast Cancer. Clin Cancer Res 24:560-568

10. Turnbull AK, Arthur LM, Renshaw L et al (2015) Accurate Prediction and Validation of Response to Endocrine Therapy in Breast Cancer. J Clin Oncol 33:2270-2278

11. Wolff AC, Hammond MEH, Allison KH et al (2018) Human Epidermal Growth Factor Receptor 2 Testing in Breast Cancer: American Society of Clinical Oncology/College of American Pathologists Clinical Practice Guideline Focused Update. J Clin Oncol 36:2105-2122

12. Ferreira MM, Ramani VC, Jeffrey SS (2016) Circulating tumor cell technologies. Mol Oncol 10:374-394

13. Sharma S, Zhuang R, Long M et al (2018) Circulating tumor cell isolation, culture, and downstream molecular analysis. Biotechnol Adv 36:1063-1078

14. Andree KC, van Dalum G, Terstappen LW (2016) Challenges in circulating tumor cell detection by the CellSearch system. Mol Oncol 10:395-407

15. Ignatiadis M, Lee M, Jeffrey SS (2015) Circulating Tumor Cells and Circulating Tumor DNA: Challenges and Opportunities on the Path to Clinical Utility. Clin Cancer Res 21:4786-4800

16. Jan YJ, Chen JF, Zhu Y et al (2018) NanoVelcro rare-cell assays for detection and characterization of circulating tumor cells. Adv Drug Deliv Rev 125:78-93 
17. Chen L, Peng M, Li N et al (2018) Combined use of EpCAM and FRa enables the high-efficiency capture of circulating tumor cells in non-small cell lung cancer. Sci Rep 8:1188

18. Gabriel MT, Calleja LR, Chalopin A et al (2016) Circulating Tumor Cells: A Review of Non-EpCAM-Based Approaches for Cell Enrichment and Isolation. Clin Chem 62:571-581

19. Varillas JI, Zhang J, Chen K et al (2019) Microfluidic Isolation of Circulating Tumor Cells and Cancer Stem-Like Cells from Patients with Pancreatic Ductal Adenocarcinoma. Theranostics 9:1417-1425

20. Liao CJ, Hsieh CH, Hung FC et al. The Integration of a Three-Dimensional Spheroid Cell Culture Operation in a Circulating Tumor Cell (CTC) Isolation and Purification Process: A Preliminary Study of the Clinical Significance and Prognostic Role of the CTCs Isolated from the Blood Samples of Head and Neck Cancer Patients. Cancers (Basel) 2019; 11

21. Qi LN, Xiang BD, Wu FX et al (2018) Circulating Tumor Cells Undergoing EMT Provide a Metric for Diagnosis and Prognosis of Patients with Hepatocellular Carcinoma. Cancer Res 78:4731-4744

22. Li C, Pan R, Li P et al (2017) Hydrogen Peroxide-Responsive Nanoprobe Assists Circulating Tumor Cell Identification and Colorectal Cancer Diagnosis. Anal Chem 89:5966-5975

23. Li C, Zhang T (2016) Human mammaglobin: A specific marker for breast cancer prognosis. J buon 21:35-41

24. Ni YB, Tsang JYS, Shao MM et al (2018) GATA-3 is superior to GCDFP-15 and mammaglobin to identify primary and metastatic breast cancer. Breast Cancer Res Treat 169:25-32

25. Chaurasiya S, Hew P, Crosley P et al (2016) Breast cancer gene therapy using an adenovirus encoding human IL-2 under control of mammaglobin promoter/enhancer sequences. Cancer Gene Ther 23:178-187

26. Pandey M, Sunil Kumar BV, Gupta K et al (2018) Over-expression of mammaglobin-B in canine mammary tumors. BMC Vet Res 14:184

27. Liang X, Shi B, Wang K et al (2016) Development of self-assembling peptide nanovesicle with bilayers for enhanced EGFR-targeted drug and gene delivery. Biomaterials 82:194-207

28. Fan M, Liang X, Li Z et al (2015) Chlorambucil gemcitabine conjugate nanomedicine for cancer therapy. Eur J Pharm Sci 79:20-26

29. Fan M, Liang X, Yang D et al (2016) Epidermal growth factor receptor-targeted peptide conjugated phospholipid micelles for doxorubicin delivery. J Drug Target 24:111-119

\section{Figures}



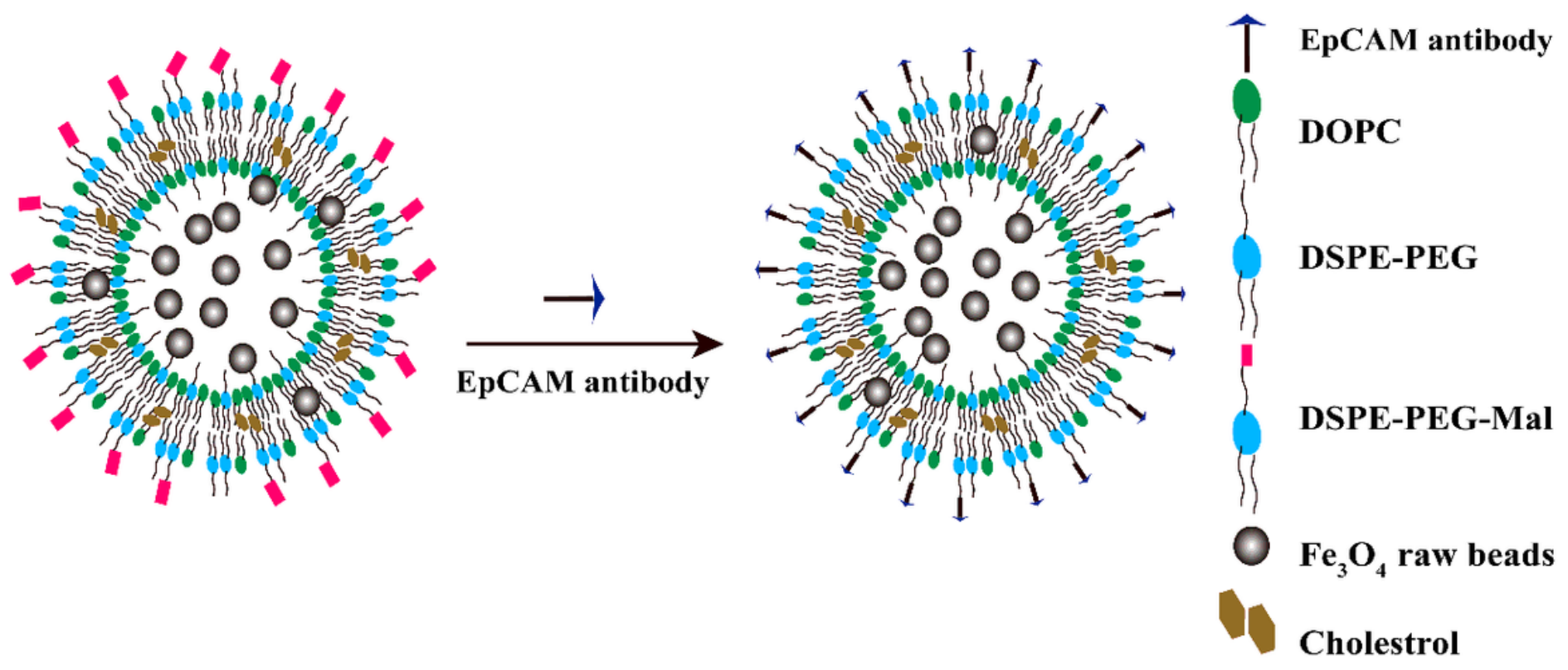

Figure 1

The preparation method of EpCAM antibody modified liposome magnetic particles (ELMP). EpCAM antibodies were modified on the surface after the preparation of the liposome magnetic particles, raw Fe304 beads were embedded inside of the liposome.
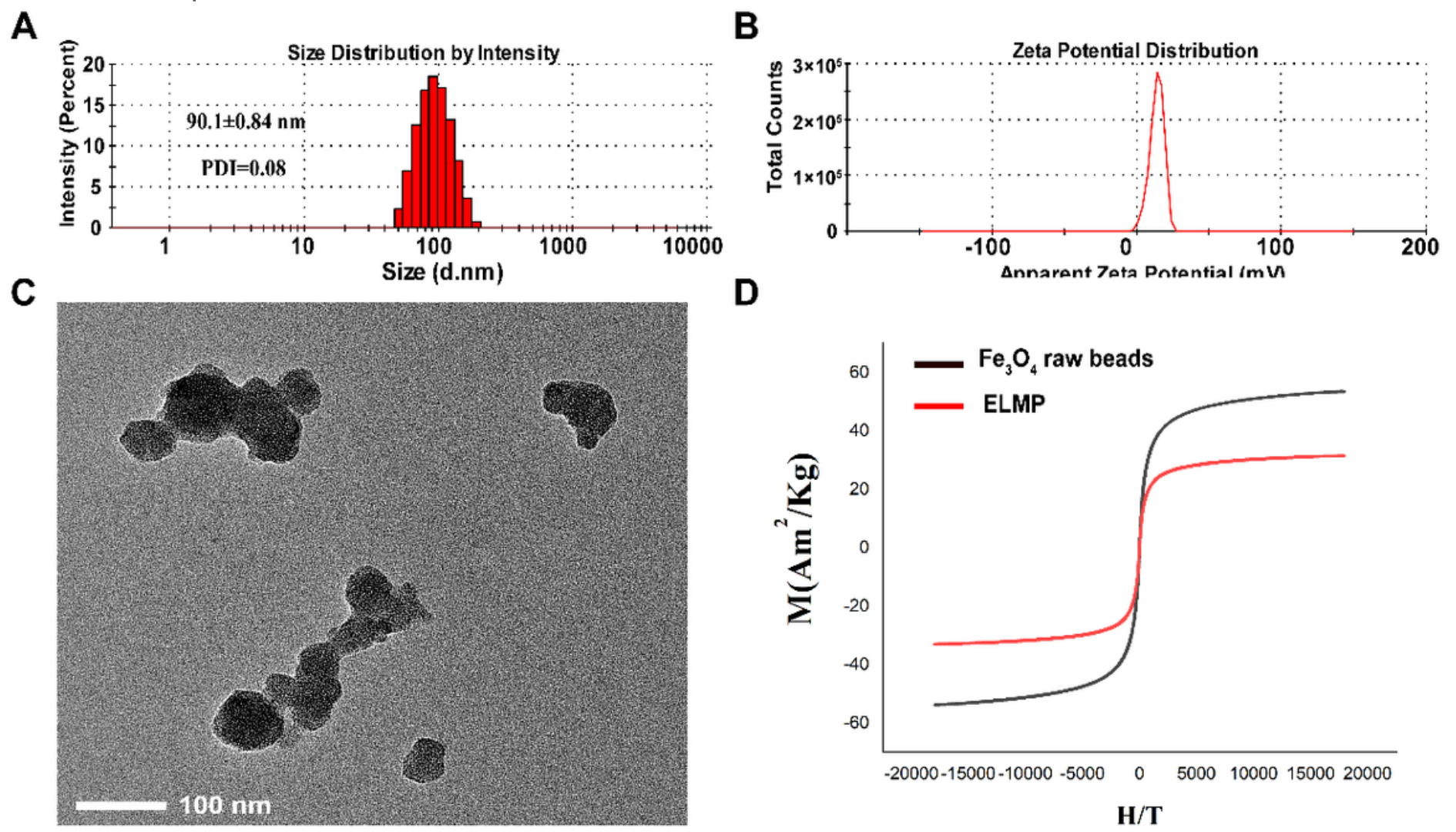

D

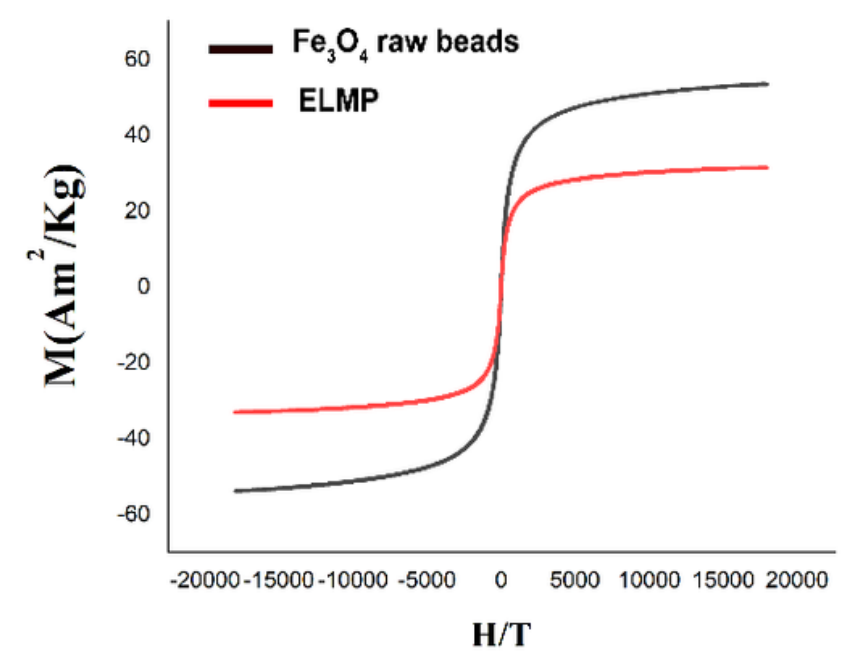

Figure 2 
Physical characterization of ELMP. (A). Size distribution of ELMP using dynamic light scattering analysis. (B). Potential distribution of ELMP. (C). Transmission Electron Microscope (TEM) observation of ELMP, scale bar is 100 nm. (D). Magnetization curve of the Fe304 raw beads and ELMP.

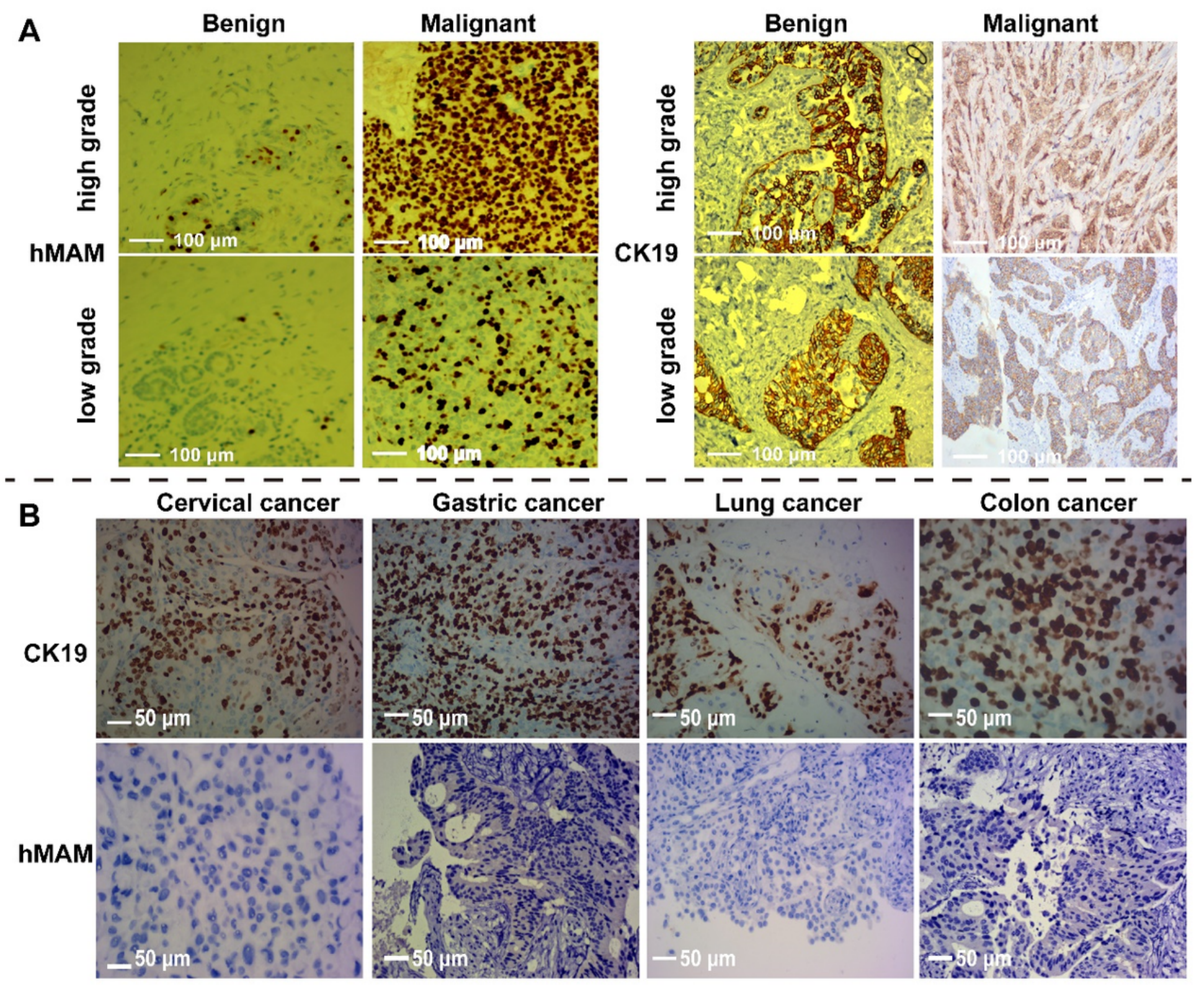

Figure 3

Immunohistochemistry of breast cancer tissue. (A). Biopsy results of mammary ducts with different degrees of malignancy. (B). Biopsy results of different malignancy cancer. 


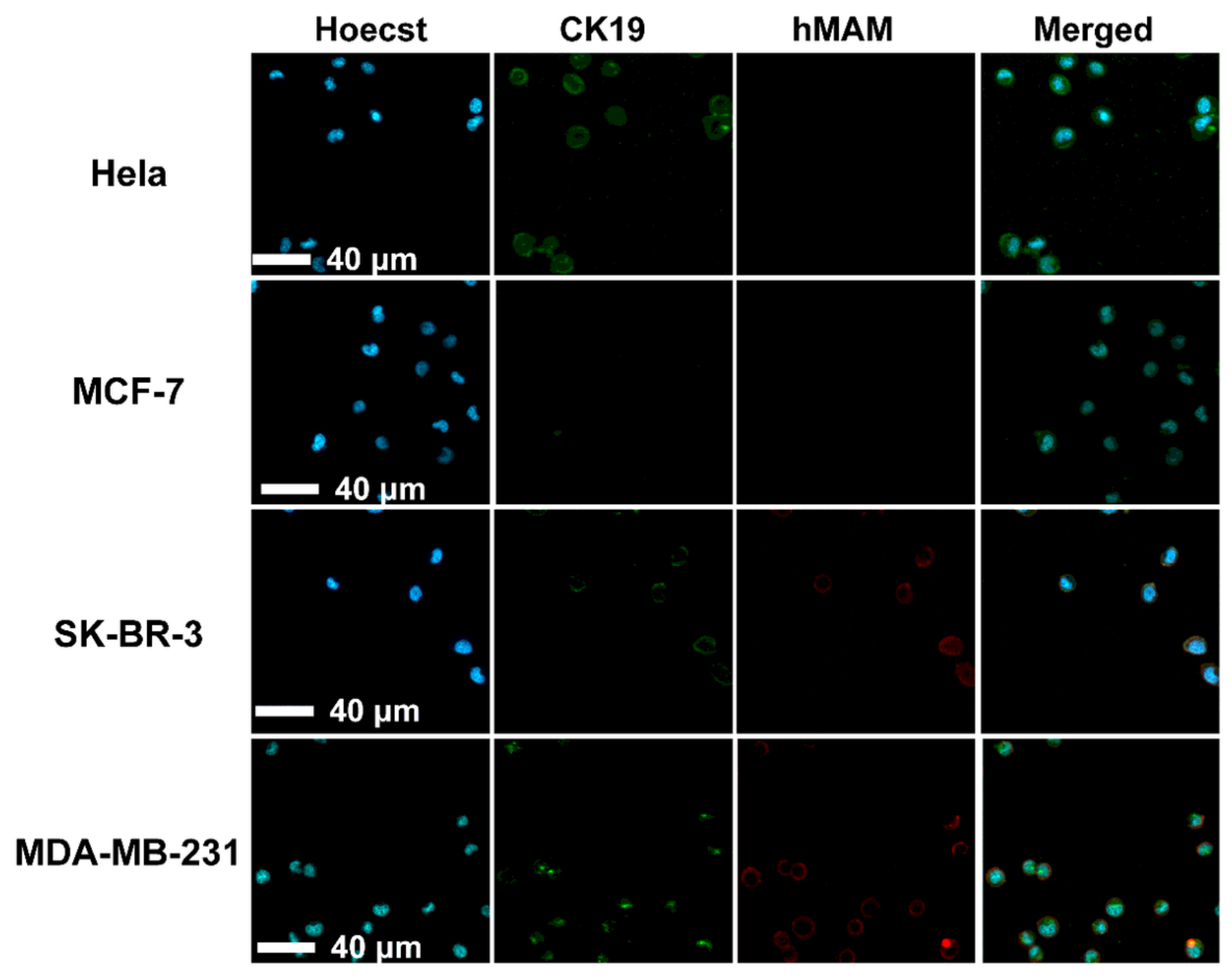

Figure 4

Confocal observation of immunofluorescence staining of cancer cells. CK19 was labeled with FITC, hMAM was labeled with QDs, and the nucleus was stained with Hoechst 33342 (the field of view is magnified 200 times). 


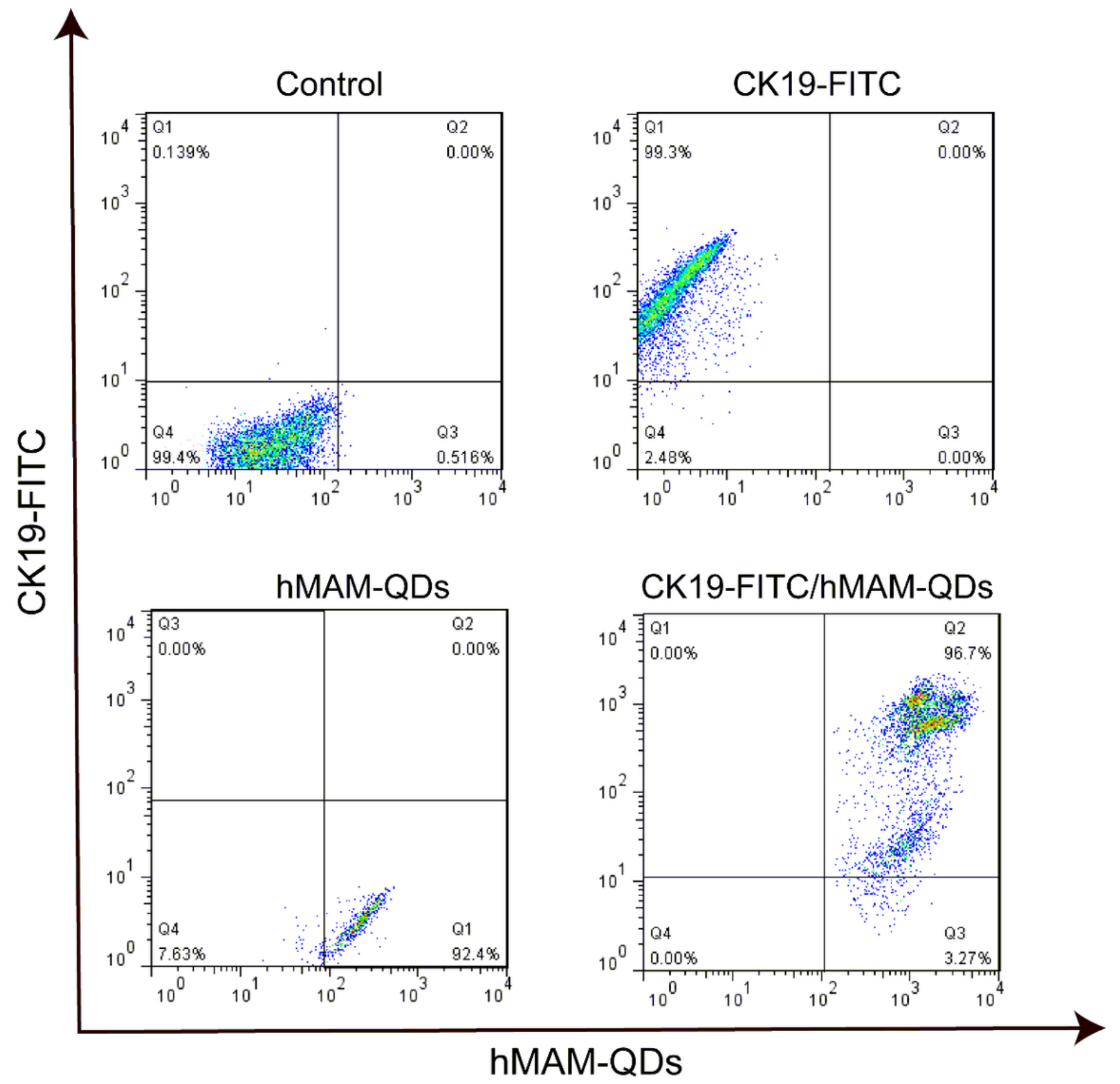

Figure 5

Flow cytometry analysis of MDA-MB-231 cells by CK19-FITC combined with hMAM-QDS staining. 


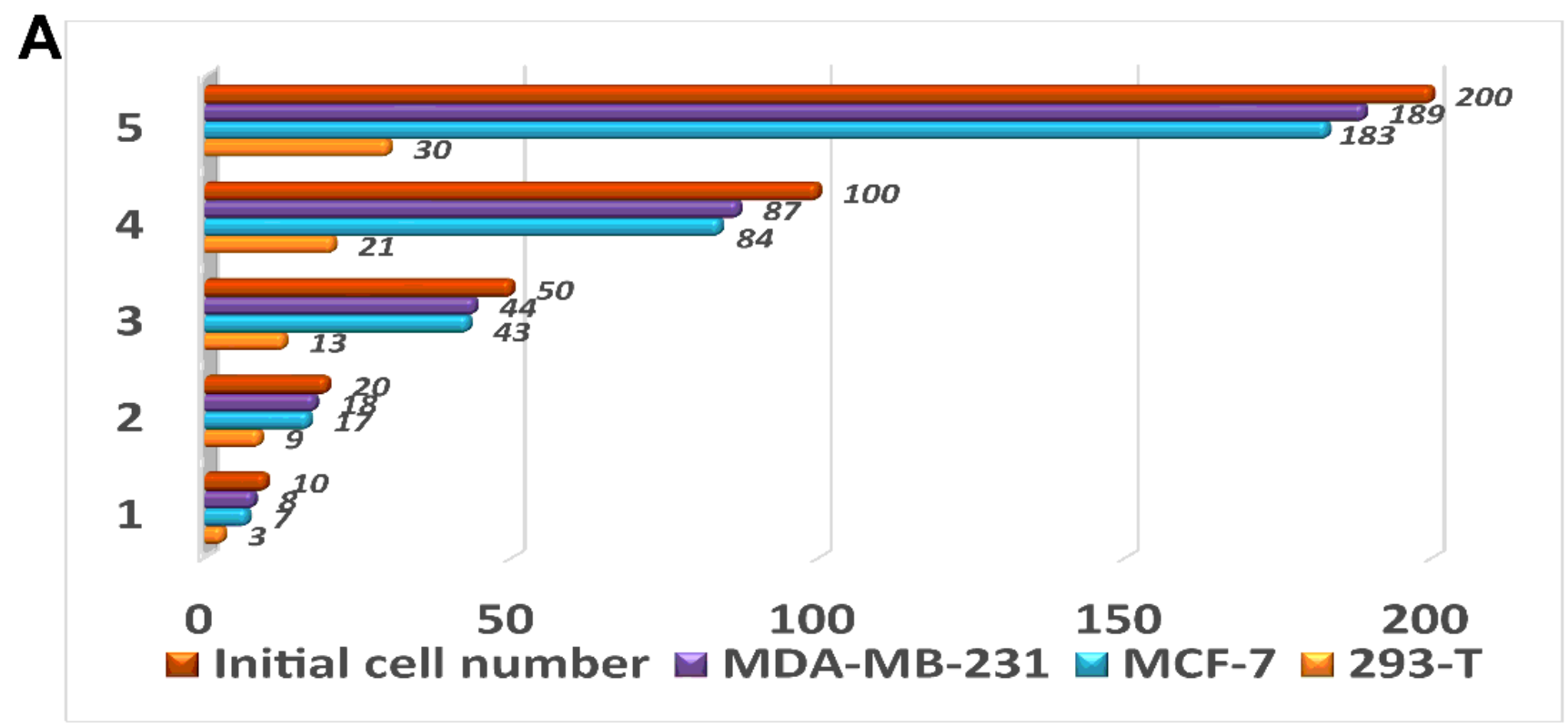

B

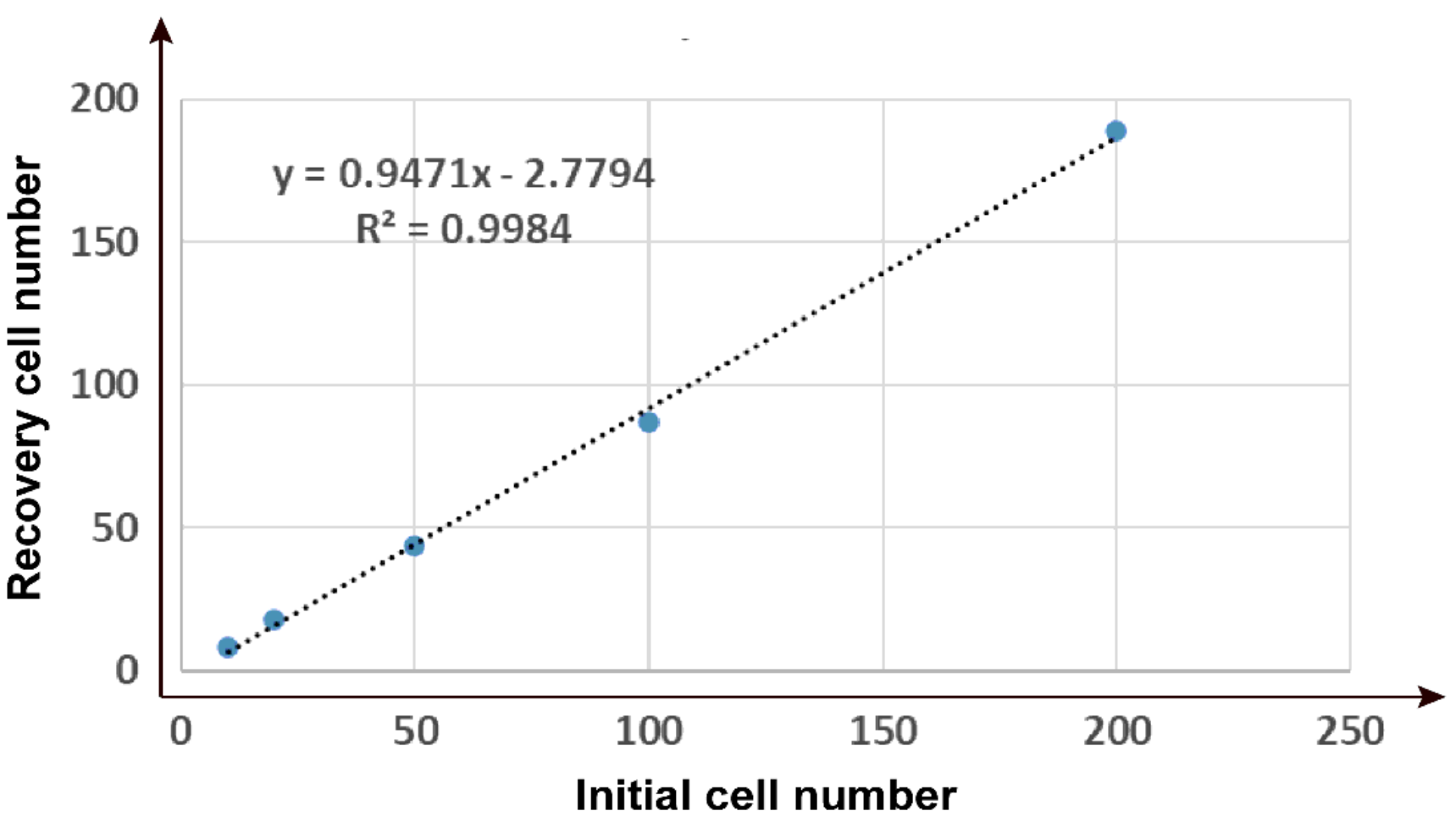

Figure 6

The MDA-MMB-231cell recycle results by using ELMP. (A). Different cancer cell recovery results in different Cell number gradient. (B). Linear analysis of MDA-MMB-231 cell recovery performance by ELMP. 


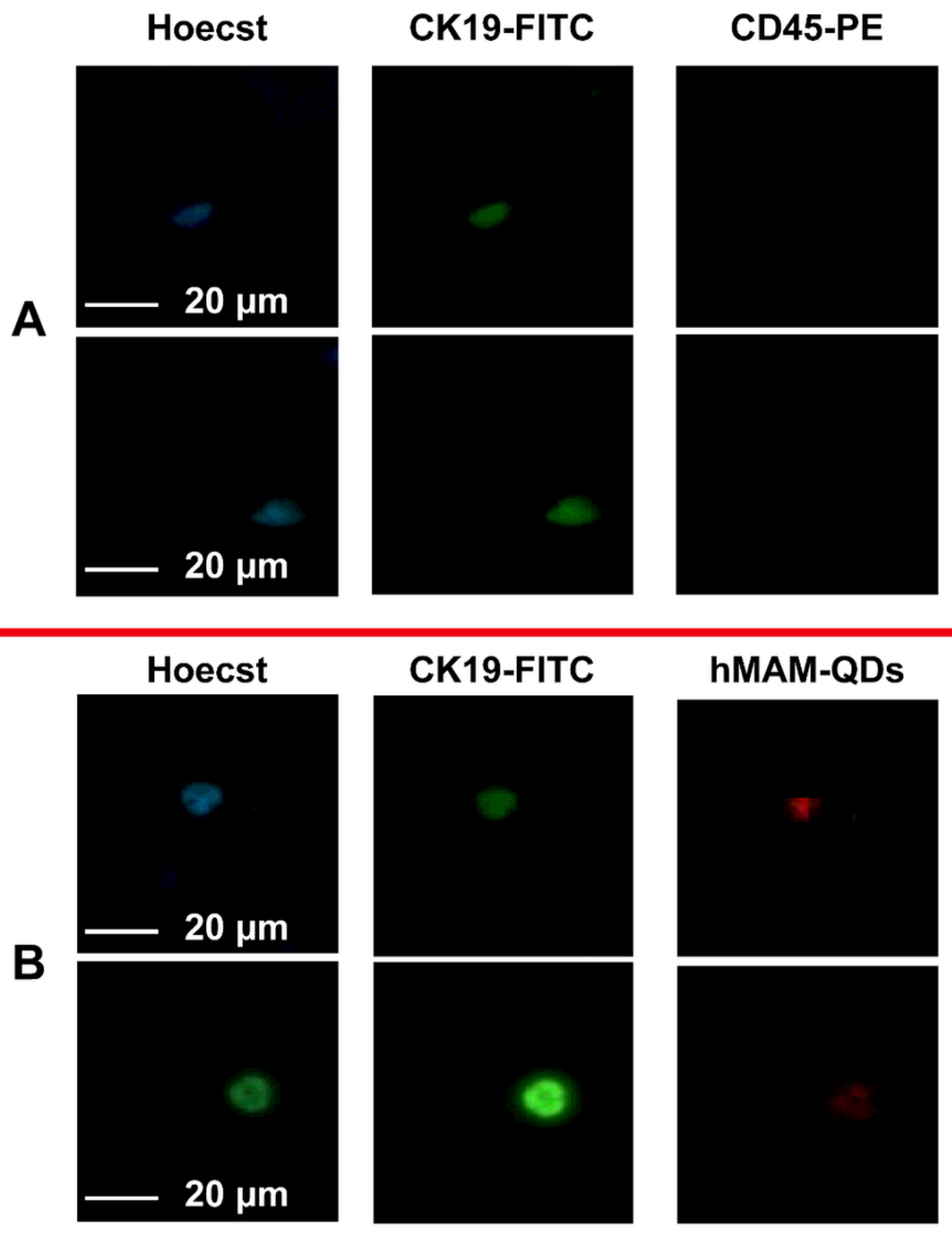

Figure 7

CTCs in blood of breast cancer patients captured by ELMP. (A). CTCs staining by single tumor marker method: CK19FITC, CD45-PE and the nucleus was stained with Hoechst 33342. (B). CTCs staining by double tumor marker method: CK19-FITC, hMAM-QDs and the nucleus was stained with Hoechst 33342 (the field of view is magnified 200 times). 


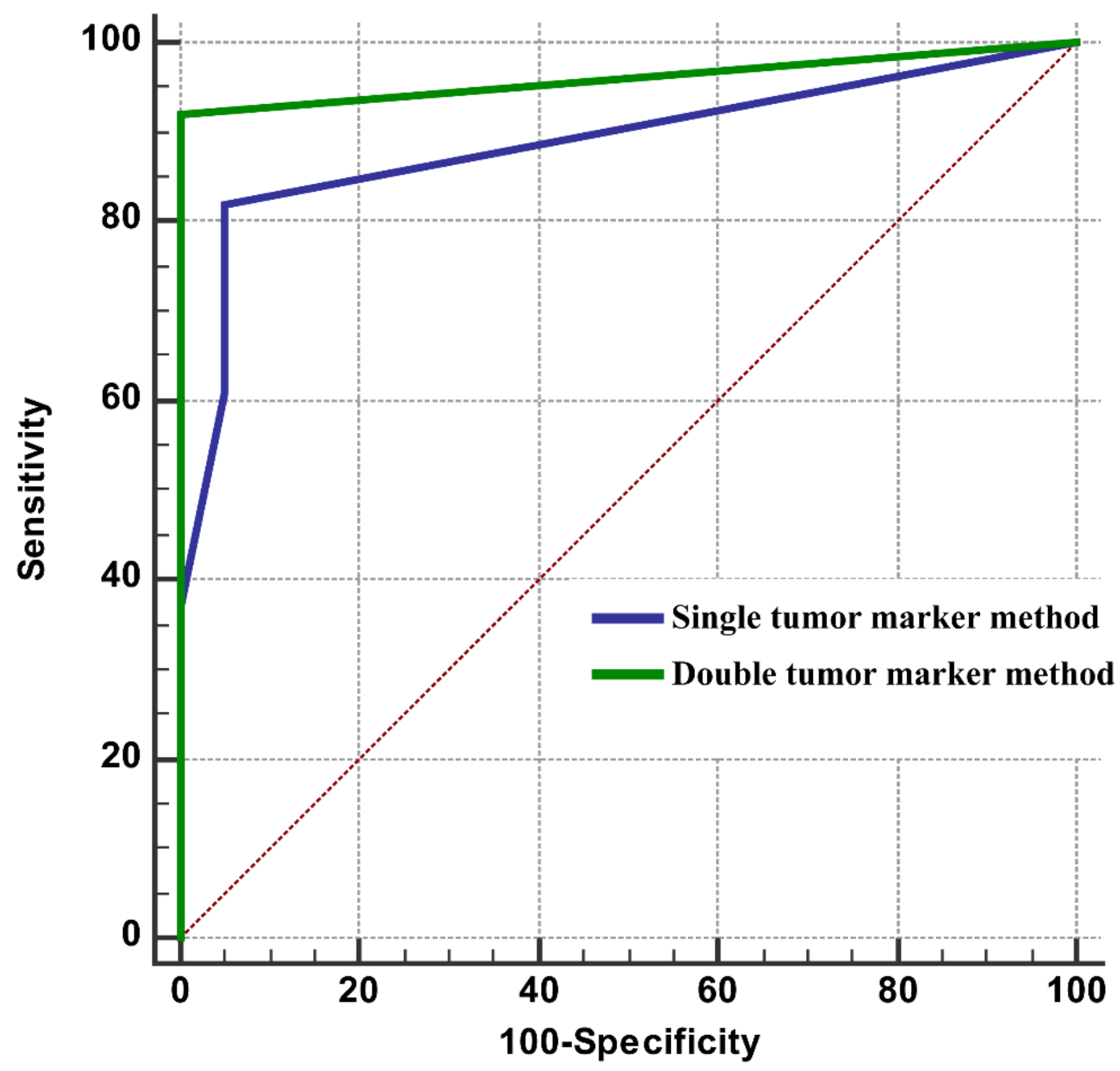

Figure 8

The ROC curve of CTC detection by Single tumor marker method and double tumor marker method. 